\title{
Melatonin and tannic acid supplementation in vitro improve fertilization and embryonic development in pigs
}

\author{
Rachel L. Lane, Brian D. Whitaker ${ }^{1}$ \\ Department of Animal and Pre-veterinary Studies, University of Findlay, Findlay OH, 45840, USA.
}

\begin{abstract}
The objective of this study was to determine the effects of melatonin supplementation during maturation and tannic acid supplementation during IVF on fertilization kinetics and early embryonic development. Experiment 1 determined the optimum concentration of melatonin supplemented to the oocytes for subsequent embryonic development. Oocytes $(n=400)$ were supplemented at $22 \mathrm{~h}$ of maturation with 0,75 , 100 , or $150 \mathrm{~nm}$ melatonin and then subjected to IVF and embryo culture. After IVF, a portion of the embryos were evaluated for penetration, polyspermy, and male pronuclear (MPN) formation rates. Embryos were evaluated $48 \mathrm{~h}$ after IVF for cleavage and $144 \mathrm{~h}$ for blastocyst formation. There were no significant differences between treatment groups with respect to penetration and polyspermy. Supplementation of $150 \mathrm{~nm}$ melatonin produced a significantly greater $(\mathrm{P}<0.05)$ percent of embryos with MPN compared to those supplemented with $75 \mathrm{~nm}$ or $100 \mathrm{~nm}$. Supplementation of $150 \mathrm{~nm}$ melatonin produced significantly less $(\mathrm{P}<0.05)$ embryos cleaved by $48 \mathrm{~h}$ after IVF while $75 \mathrm{~nm}$ melatonin supplementation had a significantly higher $(\mathrm{P}<0.05)$ percentage of blastocyst formation by $144 \mathrm{~h}$ after IVF. Based on the optimal concentration of melatonin observed in experiment 1 , experiment 2 determined the effects of supplementing $75 \mathrm{~nm}$ melatonin to the maturation media and $5.0 \mu \mathrm{g} / \mathrm{ml}$ tannic acid supplementation during IVF on oxidative stress, fertilization kinetics, and embryonic development. Oocytes $(n=720)$ were supplemented at $22 \mathrm{~h}$ of maturation with or without $75 \mathrm{~nm}$ melatonin and then fertilized with frozen-thawed sperm supplemented with or without $5 \mu \mathrm{g} / \mathrm{ml}$ tannic acid. Reactive oxygen species levels were measured in matured oocytes using 2', 7'dichlorodihydrofluorescein diacetate. Oocytes supplemented with $75 \mathrm{~nm}$ melatonin had significantly less $(\mathrm{P}<0.05)$ reactive oxygen species generation and oocytes fertilized with sperm incubated with tannic acid had a significantly less $(\mathrm{P}<0.05)$ incidence of polyspermic penetration compared to no supplementation. All treatment groups had significantly greater $(\mathrm{P}<0.05)$ incidence of male pronuclear formation compared to oocytes not supplemented with melatonin and fertilized without tannic acid. Oocytes that were supplemented with melatonin and fertilized with sperm incubated with tannic acid had a significantly higher $(\mathrm{P}<0.05)$ percentage of blastocyst formation by $144 \mathrm{~h}$ post-IVF compared all other treatment groups. Results indicate that supplementation
\end{abstract}

of $75 \mathrm{~nm}$ melatonin during oocyte maturation and 5 $\mu \mathrm{g} / \mathrm{ml}$ tannic acid during IVF leads to a decrease in oxidative stress, increase in IVF success and subsequent embryo development in pigs.

Keywords: IVF, melatonin, oocyte maturation, oxidative stress, tannic acid.

\section{Introduction}

High levels of reactive oxygen species (ROS) in and around maturing oocytes lead to oxidative stress, which hinders otherwise successful fertilization. High frequency of polyspermic penetration also presents a major obstacle to the production of in vitro derived pig embryos. Research focusing on how oocytes alleviate oxidative stress has shown the negative impact of ROS on embryonic development (Abeydeera et al., 1998; Whitaker and Knight, 2004). Melatonin is a free-radical scavenger which can cross cell membranes and the blood-brain barrier (Reiter et al., 2010; Pohanka, 2011). Additionally, melatonin interacts with other antioxidants to improve the overall effectiveness of each antioxidant (Arnao and Hernández-Ruiz, 2006). Supplementation of melatonin has shown to lower ROS levels during oocyte maturation in mice (Salehi et al., 2014), bovine (Cebrian-Serrano et al., 2013) and humans (Wei et al., 2013). In pigs, melatonin his been shown to support oocyte maturation and embryo culture (Do et al., 1015) and to protect the oocyte against ROS (Li et al., 2015).

Polyspermic penetration in porcine oocytes is still a major challenge for researchers and remains around $30 \%$ in most laboratories (Abedyeera et al., 1998; Fan and Sun, 2004). Supplementation of tannic acid during in vitro fertilization (IVF) reduces polyspermy in porcine oocytes by inhibiting hyaluronidase activity, thus reducing polyspermic penetration (Tatemoto et al., 2006).

To our knowledge, research has not been published focusing on the supplementation of both melatonin to porcine oocytes during maturation and tannic acid to frozen-thawed boar spermatozoa during IVF. Therefore, the objective of this study was to determine the effects of melatonin supplementation to the pig oocyte maturation media and $5 \mu \mathrm{g} / \mathrm{ml}$ tannic acid to the IVF media on oxidative stress, IVF kinetics and embryonic development. The production of ROS was measured in matured oocytes to determine oxidative stress levels. Oocyte fertilization, polyspermy, and male pronuclear (MPN) formation were observed to determine the IVF kinetics. Embryos were evaluated for cleavage and blastocyst formation. 


\section{Materials and Methods}

Media

Unless otherwise stated, all chemicals were purchased from Sigma-Aldrich Co. (St. Louis, MO, USA). The oocyte maturation media was Medium 199 (M199) with Earle's salts (Fisher Scientific, Pittsburgh, PA, USA) supplemented with $5 \mu \mathrm{g} / \mathrm{ml}$ follicle stimulating hormone (FSH), $1 \mu \mathrm{l} / \mathrm{ml}$ insulin, $50 \mathrm{ng} / \mathrm{ml}$ gentamicin sulfate, $10 \mathrm{ng} / \mathrm{ml}$ epidermal growth factor, and $10 \%$ fetal calf serum (v/v; FCS). The IVF medium used was a modified Tris-buffered media formulated by Abeydeera and Day (1997). The embryo culture medium used was North Carolina State University (NCSU) 23 medium (Petters and Wells, 1993) containing $0.4 \%(\mathrm{w} / \mathrm{v})$ bovine serum albumin (BSA). All media were filtered through a $0.22 \mu \mathrm{m}$ pore MCE membrane (Fisher Scientific, Pittsburgh PA, USA) syringe filter. All incubations were carried out under mineral oil at $38.5^{\circ} \mathrm{C}$ in an atmosphere of $5 \% \mathrm{CO} 2$ unless otherwise indicated.

\section{Maturation of oocytes}

Oocytes were aspirated from mature follicles (3-6 mm diameter) obtained from adult crossbred sows (at least 18 months of age) at a local abattoir. The average elapsed time between ovary collection and follicular aspiration was $3 \mathrm{~h}$. Oocytes surrounded by a compact cumulus cell mass and uniform ooplasm were washed three times and placed (45-55 oocytes/well) into $500 \mu \mathrm{l}$ of maturation medium. After 20-24 h from initial placement in media, oocytes were washed three times in maturation media and placed (45-55 oocytes/well) into $500 \mu \mathrm{l}$ of maturation media without FSH and FCS for an additional 18-26 h. After incubation, cumulus cells were removed from the oocytes by repeat pipetting in M199 containing $0.1 \%$ hyaluronidase (w/v). Only oocytes observed with uniform granulated cytoplasm and an extruded polar body were washed in IVF medium and used as described below.

\section{Measuring ROS production}

Levels of ROS in matured oocytes were measured by incubating the oocytes in $0.3 \%(\mathrm{w} / \mathrm{v}) \mathrm{BSA}$ in PBS with $5 \mu \mathrm{m} \mathrm{2',7'-dichlorodihydrofluorescein}$ diacetate (DCHF-DA) for $30 \mathrm{~min}$. Oocytes were then examined using fluorescent microscopy (excitation maximum wavelength $=490 \mathrm{~nm}$ and emission maximum wavelength $520=\mathrm{nm}$ ), their images were recorded digitally and the fluorescence brightness at the equatorial section of each oocyte stained by DCHF-DA was calculated using computer software (Nikon NIS Elements; Nikon Instruments Inc., Melville, NY, USA). The data were presented as the percentage of fluorescent intensity present in the oocytes matured without supplementation.

\section{Spermatozoa preparation}

Three frozen semen pellets, one from each of three different boars (International Boar Semen, Eldora,
IA, USA) were thawed in IVF media and centrifuged at $36.3 \mathrm{x} g$ for $5 \mathrm{~min}$. The semen was then washed twice at $553 \times \mathrm{g}$ for $5 \mathrm{~min}$. After washing, the spermatozoa pellet was re-suspended in IVF media at a concentration of 2.0 x $10^{5}$ sperm cells $/ \mathrm{ml}$ and incubated for $1 \mathrm{~h}$ before $50 \mu \mathrm{l}$ was added to each group of oocytes in $50 \mu \mathrm{l}$ droplets of IVF medium. Immediately prior to IVF, sperm were analyzed for forward progressive motility using a phasecontrast microscope at $400 \mathrm{X}$ magnification and their viability/membrane integrity was assessed by staining with $0.6 \%$ Eosin red (w/v) and 5.0\% Aniline blue (w/v) dye to ensure a quality sample of sperm being used for IVF. The frozen-thawed sperm samples used averaged $64.8 \pm 5.0 \%$ forward progressive motility and $70.9 \pm 5.0 \%$ live.

After 4-6 h of IVF, the putative zygotes were washed three times and placed (50 zygotes per well) into $500 \mu \mathrm{l}$ of embryo culture medium and incubated. Embryos were evaluated for cleavage and blastocyst formation under a stereomicroscope at 48 and $144 \mathrm{~h}$ after IVF, respectively.

\section{Examination of IVF characteristics}

Approximately $12 \mathrm{~h}$ after IVF, oocytes were mounted and fixed with $25 \%$ acetic acid in ethanol $(\mathrm{v} / \mathrm{v})$ at room temperature. After $48 \mathrm{~h}$ of fixation, oocytes were stained with $1 \%$ orcein $(\mathrm{w} / \mathrm{v})$ in $45 \%$ acetic acid $(\mathrm{v} / \mathrm{v})$ and examined using a phase-contrast microscope at $400 \mathrm{X}$ magnification. Oocytes were characterized by visualization of penetration, MPN formation, and polyspermic penetration. Oocytes were considered penetrated when they had one or more swollen sperm head(s) or MPN and their corresponding sperm tails.

\section{Experimental design}

\section{Experiment 1: determination of optimum concentration of melatonin for embryo production}

Oocytes were matured in maturation media for 20-24 $\mathrm{h}$ and then in fresh maturation media without FCS and hormones for an additional 18-26 h. During the second stage of maturation, the fresh media was supplemented with $0,75,100$ or $150 \mathrm{~nm}$ melatonin. After IVF, the number of oocytes penetrated was recorded ( $\mathrm{n}=200 ; 50$ oocytes/treatment group). Of those penetrated, the number of polyspermic oocytes, and the number of oocytes penetrated with a MPN were determined. During embryonic development $(n=400$; 100 oocytes per treatment group), the number of embryos cleaved at $48 \mathrm{~h}$ after IVF and blastocysts at $144 \mathrm{~h}$ after IVF were observed and recorded. A total of 600 matured oocytes (150 oocytes/treatment group) over two replicates were used in this experiment.

\section{Experiment 2: effects of melatonin and tannic acid supplementation on IVF and embryonic development}

Experiment two supplemented $75 \mathrm{~nm}$ melatonin to the maturation media, since it elicited the lowest rate of polyspermic penetration and highest penetration rate, MPN formation and cleavage and blastocyst formation rate. The effects of supplementing 
$75 \mathrm{~nm}$ melatonin to the maturation media during the later stages of oocyte maturation (from 22 to $48 \mathrm{~h}$ ) on the levels of ROS in matured oocytes were measured by DCHF-DA staining at the end of maturation $(n=120$; 60 oocytes/treatment group). Additional maturated oocytes were subjected to IVF with the fertilization media supplemented with $5.0 \mu \mathrm{g} / \mathrm{ml}$ tannic acid Endpoints measured were the number of oocytes penetrated, the number of polyspermic oocytes (of those penetrated), and the number of oocytes penetrated with a MPN (of those penetrated; $\mathrm{n}=200 ; 50$ oocytes/treatment group). During embryonic development $(n=400 ; 100$ oocytes/treatment group), the number of embryos cleaved at $48 \mathrm{~h}$ after IVF and blastocysts at $144 \mathrm{~h}$ after IVF were observed and recorded. A total of 720 matured oocytes (180 oocytes/treatment group) over three replicates were used in this experiment.

\section{Statistical analysis}

Data were analyzed by one-way ANOVA using the PROC GLM procedures of SAS (SAS Institute, Cary, $\mathrm{NC}$, USA). When there was a significant effect, significant differences were determined using the LSMEANS statement and Tukey adjustment for multiple comparisons. The effects included in the initial model were treatment, well and replicate. Well and replicate effects were not significant $(\mathrm{P}>0.05)$ and were deleted from the finals models. Chi-square analysis was used to determine percentages of embryos reaching the different developmental stages for each treatment. In all analyses, $\mathrm{P}<0.05$ was considered to be significant. Results are expressed as the least-squares mean $\pm \mathrm{SEM}$.

\section{Results}

Experiment 1: determination of optimum concentration of melatonin for embryo production

Oocytes supplemented with $75 \mathrm{~nm}$ melatonin had significantly lower $(\mathrm{P}<0.05)$ incidences of polyspermic penetration $(13.75 \pm 3.11 \%)$ compared to all other treatment groups and significantly higher $(\mathrm{P}<0.05) \mathrm{MPN}$ formation $(66.25 \pm 9.29 \%)$ compared to oocytes supplemented with $150 \mathrm{~nm}$ melatonin (27.50 $\pm 10.84 \%$; Table 1 ).

Embryo development results are shown in Fig. 1. There were no differences in the percentage of embryos cleaved by $48 \mathrm{~h}$ between no melatonin supplementation, $75 \mathrm{~nm}$ or $100 \mathrm{~nm}$ supplementations. Oocytes supplemented with $150 \mathrm{~nm}$ melatonin had a significantly fewer $(\mathrm{P}<0.05)$ percentage of embryos cleaving by $48 \mathrm{~h}$ after IVF $(22.00 \pm 21.37 \%)$ compared to all other treatment groups. Oocytes supplemented with $75 \mathrm{~nm}$ melatonin had a significantly higher $(\mathrm{P}<0.05)$ percentage of embryos reaching the blastocyst stage of development by $144 \mathrm{~h}$ after IVF $(32.00 \pm 17.32 \%)$ compared to oocytes supplemented with $150 \mathrm{~nm}$ melatonin $(10.00 \pm 17.32 \%)$.

Table 1. Effects of melatonin supplementation on oocyte fertilization characteristics $12 \mathrm{~h}$ after fertilization.

\begin{tabular}{lccc}
\hline Treatment group & Oocytes penetrated $(\%)$ & Polyspermic oocytes $^{\S}(\%)$ & Oocytes with MPN $^{\S}(\%)$ \\
\hline No melatonin & $86.00 \pm 8.18$ & $25.58 \pm 8.72^{\mathrm{a}}$ & $47.67 \pm 9.34^{\mathrm{ac}}$ \\
$75 \mathrm{~nm}$ melatonin & $80.00 \pm 8.18$ & $13.75 \pm 3.11^{\mathrm{b}}$ & $66.25 \pm 9.29^{\mathrm{ab}}$ \\
$100 \mathrm{~nm}$ melatonin & $80.00 \pm 8.18$ & $30.00 \pm 7.14^{\mathrm{a}}$ & $80.00 \pm 4.54^{\mathrm{b}}$ \\
$150 \mathrm{~nm}$ melatonin & $85.00 \pm 8.18$ & $22.35 \pm 4.33^{\mathrm{a}}$ & $27.50 \pm 10.84^{\mathrm{c}}$ \\
\hline
\end{tabular}

${ }^{\dagger}$ Treatment groups were the final concentration of melatonin supplemented to the oocyte maturation media in the second phase of maturation. ${ }^{\S}$ Percentage of the number of oocytes penetrated. ${ }^{\mathrm{a}, \mathrm{b}}$ Means within a column with different superscripts differ significantly $(\mathrm{P}<0.05)$. Differences between columns are not comparable. Data are expressed as Mean \pm SEM.

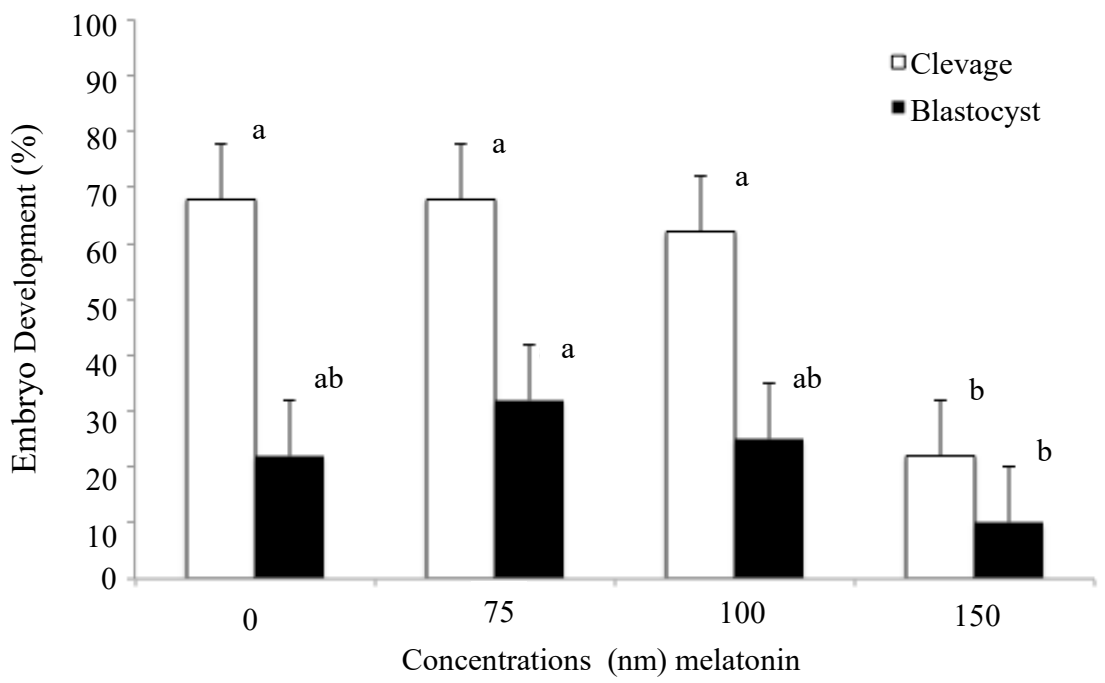

Figure 1. Effects of different melatonin concentrations supplemented in the oocyte maturation media at $22 \mathrm{~h}$ on embryo development $(\mathrm{n}=400)$ in experiment 1 . Cleavage, observed $48 \mathrm{~h}$ after IVF; blastocysts, observed $144 \mathrm{~h}$ after IVF. ${ }^{\mathrm{a}, \mathrm{b}}$ Means with different superscripts differ at least $\mathrm{P}<0.05$. Differences between cleavage and blastocyst are not comparable. Data expressed as Mean \pm SEM. 
Experiment 2: effects of melatonin and tannic acid supplementation on IVF and embryonic development

Supplementation of $75 \mathrm{~nm}$ of melatonin was the lowest supplementation level that did not have detrimental effects on sperm penetration, MPN formation, cleavage and blastocyst formation and thus was the supplementation level used in experiment 2.

Oocytes supplemented with $75 \mathrm{~nm}$ melatonin significantly decreased $(\mathrm{P}<0.05)$ ROS generation $(67.00 \pm 3.02 \%)$ compared to oocytes with no melatonin supplementation $(100 \pm 3.02 \%)$. There were no significant differences in penetration rates of the oocytes however oocytes fertilized with sperm incubated with tannic acid had a significantly less ( $\mathrm{P}<$ 0.05) incidence of polyspermic penetration. All treatment groups had significantly greater $(\mathrm{P}<0.05)$ incidence of male pronuclear formation compared to oocytes not supplemented with melatonin and fertilized without tannic acid (Table 2).

There were significant differences $(\mathrm{P}<0.05)$ in cleavage rates by $48 \mathrm{~h}$ post-IVF between each of the groups (Fig. 2). Oocytes supplemented with melatonin and fertilized with sperm incubated with tannic acid had a significantly higher $(\mathrm{P}<0.05)$ percentage of blastocyst formation by $144 \mathrm{~h}$ post-IVF $(48 \pm 4.04 \%)$ compared all other treatment groups (Fig. 2).

Table 2. Effects of melatonin and tannic acid supplementation on oocyte fertilization characteristics $12 \mathrm{~h}$ after fertilization.

\begin{tabular}{lccc}
\multicolumn{1}{c}{ Treatment group } & \\
& $\begin{array}{c}\text { Oocytes penetrated } \\
(\%)\end{array}$ & $\begin{array}{c}\text { Polyspermic oocytes } \\
(\%)\end{array}$ & $\begin{array}{c}\text { Oocytes with MPN }^{\S} \\
(\%)\end{array}$ \\
\hline No melatonin, no tannic acid & $84.00 \pm 3.50$ & $33.33 \pm 2.10^{\mathrm{a}}$ & $66.67 \pm 5.51^{\mathrm{a}}$ \\
$75 \mathrm{~nm}$ melatonin, no tannic acid & $80.00 \pm 5.30$ & $27.50 \pm 4.11^{\mathrm{a}}$ & $82.50 \pm 4.37^{\mathrm{b}}$ \\
No melatonin, $5.0 \mu \mathrm{g} / \mathrm{ml}$ tannic acid & $90.00 \pm 5.58$ & $17.78 \pm 4.09^{\mathrm{b}}$ & $84.44 \pm 3.53^{\mathrm{b}}$ \\
$75 \mathrm{~nm}$ melatonin, $5.0 \mu \mathrm{g} / \mathrm{ml}$ tannic acid & $84.00 \pm 2.00$ & $16.67 \pm 5.85^{\mathrm{b}}$ & $85.71 \pm 4.35^{\mathrm{b}}$ \\
\hline
\end{tabular}

${ }^{\dagger}$ Treatment groups were the final concentration of melatonin supplemented to the oocyte maturation media in the second phase of maturation, and the final concentration of tannic acid supplemented to the IVF media during sperm thawing and IVF. ${ }^{\S}$ Percentage of the number of oocytes penetrated. ${ }^{\mathrm{a}, \mathrm{b}}$ Means within a column with different superscripts differ significantly $(\mathrm{P}<0.05)$. Differences between columns are not comparable. Data are expressed as Mean \pm SEM.

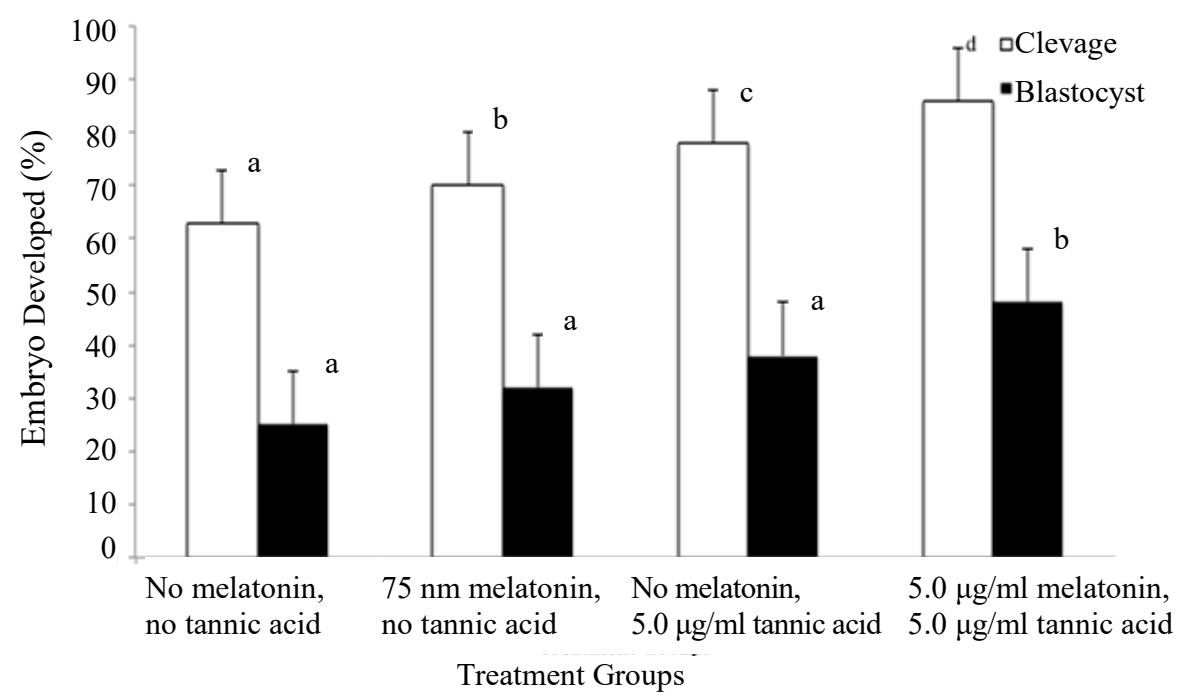

Figure 2. Effects of $75 \mathrm{~nm}$ melatonin supplemented in the oocyte maturation media at $22 \mathrm{~h}$ and $5.0 \mu \mathrm{g} / \mathrm{ml}$ tannic acid supplemented to the IVF media during sperm thawing and IVF on embryo development $(\mathrm{n}=400)$ in experiment 2. Cleavage, observed $48 \mathrm{~h}$ after IVF; blastocysts, observed $144 \mathrm{~h}$ after IVF. ${ }^{\mathrm{a}, \mathrm{b}}$ Means with different superscripts differ at least $\mathrm{P}<0.05$. Differences between cleavage and blastocyst are not comparable. Data expressed as Mean $\pm \mathrm{SEM}$.

\section{Discussion}

Despite continual advancements, IVF of porcine oocytes continues to be a challenge owing to poor cytoplasmic maturation, high incidences of polyspermic penetration, and elevated levels oxidative stress during maturation, which often leads to higher levels of ROS production (Abeydeera, 2002; Gil et al., 2010; Dang-Nguyen et al., 2011, Alvarez et al., 2015). Embryonic development is impacted by the quality of the spermatozoa used in fertilization as well as environmental conditions (Wang et al., 2003; Gil et al., 2008). Pigs typically have a high amount of oxygen tension that is present during in vitro maturation, which accumulate ROS inside the oocyte and cause damage to the DNA, which impairs embryo development (Kitagawa et al., 2004).

Previous research has indicated that the supplementation of antioxidants such as melatonin may decrease ROS and improve subsequent embryonic 
development in multiple species (Cebrian-Serrano et al., 2013;Salehi et al., 2014). Additionally, melatonin has been shown to exist in the follicular fluid surrounding pig oocytes (Shi et al., 2009). Supplemention $1 \mathrm{~nm}$ melatonin during oocyte maturation and embryo culture improved cleavage and blastocyst formation rates in vitro (Shi et al., 2009). In agreement with those studies, our results indicated that supplementing melatonin during oocyte maturation improved early embryonic development rates.

Melatonin has also been shown to reduce levels of ROS and enhance glutathione production and decrease apoptosis in pig oocytes (Li et al., 2015). Our results agree with this study, as we found supplementation of melatonin to decrease the ROS produced during maturation. The level of melatonin appears to impact the success of oocyte maturation, as a previous study reported minimal improvements, however the levels were lower than those used in the current study (Choe et al., 2010). Shi et al. (2009) reported that the concentrations of melatonin change as the follicle changes size, suggesting an effect of melatonin in oocyte maturation: as the follicle size increased, the levels of melatonin decreased. In addition to alleviating oxidative stress in oocytes, Jang et al. (2010) demonstrated that when melatonin was supplemented to boar semen, semen characteristics and developmental quality of in vitro derived embryos improved.

Supplementation of $5 \mu \mathrm{g} / \mathrm{ml}$ tannic acid to the sperm thawing and fertilization media decreases polyspermic penetration in porcine oocytes (Tatemoto et al., 2006). Tannic acid has antihyaluronidase activity and scavenges ROS in boar sperm, which has shown to improve IVF in pigs (Tokeshi et al., 2007). Our results were similar, as supplementation of tannic acid to the fertilization media decreases polyspermic penetration regardless if melatonin was supplemented during maturation. Matured oocytes supplemented with $75 \mathrm{~nm}$ melatonin and fertilized in media containing of $5 \mu \mathrm{g} / \mathrm{ml}$ tannic acid had significantly higher cleavage rates at 48 $\mathrm{h}$ post-IVF and blastocyst formation by $144 \mathrm{~h}$ post-IVF than no supplementations or supplementations of only melatonin or tannic acid.

Although our results and Tatemoto's et al. (2006) indicate that tannic acid supplementation during IVF reduces polyspermic penetration in pigs, more studies should be conducted to determine if supplementing tannic acid during oocyte maturation or during the freezing process of semen has the ability to further improve fertilization success and embryonic development. Varying the concentration of tannic acid during IVF could change the success rates (Li et al., 1997) so further studies should be conducted to determine if there is species or even boar variability. Melatonin supplementation is currently a popular topic of interest and appears to improve multiple mechanisms in the oocyte, including meiotic maturation (Park et al., 2017), embryonic development (Chen et al., 2017), and lipid metabolism (He et al., 2018). Despite the promising effects of melatonin supplementation, its specific mechanism(s) of action on the pig oocyte are relatively unknown. More research needs to be done to elucidate how melatonin affects the oocyte and if there is an endogenous role or it is entirely a supplemental effect.

Since the continual production of high quality porcine embryos has enumerable benefits, it would be advantageous for the development of a chemically defined in vitro system. Notwithstanding extensive research, polyspermic penetration is still a major issue in IVF for the porcine species, which is a critical component in the process of development. To our knowledge, this is the first study to provide information on the effects of melatonin supplementation to the maturation media with tannic acid supplementation to the fertilization media of pig oocytes. Our results indicate that supplementation with melatonin and tannic acid have beneficial effects on reducing polyspermic penetration and improving early embryonic development. Further work needs to be conducted to determine the specific mechanisms of action of melatonin on the oocyte and its surrounding environment. These data will improve our knowledge of alleviating polyspermy associated with IVF and could be used to develop new methods to modify the IVF conditions to improve the in vitro production of pig embryos.

\section{References}

Abeydeera LR, Day BN. 1997. In vitro penetration of pig oocytes in a modified Tris-buffered medium: effect of BSA, caffeine and calcium. Theriogenology, 48:537544.

Abeydeera LR, Wang WH, Cantley TC, Prather RS, Day BN. 1998. Presence of $\beta$-mercaptoethanol can increase the glutathione content of pig oocytes matured in vitro and the rate of blastocysts development after in vitro fertilization. Theriogenology, 50:747-756

Abeydeera LR. 2002. In vitro production of embryos in swine. Theriogenology, 57:256-273.

Alvarez GM, Morado SA, Soto MP, Dalvit GC, Cetica PD. 2015. The control of reactive oxygen species influences porcine oocyte in vitro maturation. Reprod Domest Anim, 50:200-205.

Arnao MB, Hernández-Ruiz J. 2006. The physiological function of melatonin in plants. Plant Signal Behav, 1:89-95.

Cebrian-Serrano A, Salvador I, Raga E, Dinnyes A, Silvestre MA. 2013. Beneficial effect of melatonin on blastocyst in vitro production from heat-stressed bovine oocytes. Reprod Domest Anim, 48:738-746.

Chenn Z, Zuo X, Hong R, Ding B, Liu C, Gao D, Shang H, Cao Z, Huang W, Zhang X, Zhang $Y$. 2017. Effects of melatonin on maturation, histone acetylation, autophagy or porcine oocyte and subsequent embryonic development. Anim Sci J, 88:1298-1310.

Choe, C, Shin YW, Kim EJ, Cho SR, Kim HJ, Choi, SH, Han MH, Han J, Son DS, Kang D. 2010. Synergistic effects of glutathione and Bmercaptoethanol treatment during in vitro maturation or porcine oocytes on early embryonic development in a culture system supplemented with L-cysteine. $J$ Reprod Dev, 56:575-582.

Dang-Nguyen TQ, Somfai T, Haraguchi S, Kikuchi 
K, Tajima A, Kanai Y, Nagai T. 2011. In vitro production of porcine embryos: current status, future perspectives and alternative applications. Anim Sci J, 82:374-382.

Do LTK, Shibata Y, Taniguchi M, Nii M, Nguyen TV, Tanihara F, Takagi M, Otoi T. 2015. Melatonin supplementation during in vitro maturation and development supports the development of porcine embryos. Reprod Domest Anim, 45:40-48.

Fan H, Sun Q. 2004. In vitro maturation and fertilization of pig oocytes. Methods Mol Biol, 253:227-233.

Gil MA, Alminana C, Roca J, Vazquez JM, Martinez EA. 2008. Boar semen variability and its effects on IVF efficiency. Theriogenology, 70:1260-1268.

Gil MA, Cuello C, Parrilla I, Vazquez JM, Roca J, Martinez EA. 2010. Advances in swine in vitro embryo production technologies. Reprod Domest Anim, 45:40-48.

He B, Yin C, Gong Y, Liu J, Guo H, Zhao R. 2018. Melatonin-induced increase of lipid droplets accumulation and in vitro maturation in porcine oocytes is mediated by mitochondrial quiescence. $J$ Cell Physiology, 233:302-312.

Jang HY, Kim YH, Kim BW, Park IC, Cheong HT, Kim JT, Park CK, Kong HS, Lee HK, Yang BK. 2010. Ameliorative effects of melatonin against hydrogen peroxide-induced oxidative stress on boar sperm characteristics and subsequent in vitro embryo development. Reprod Domest Anim, 45:943-950.

Kitagawa Y, Suzuki K, Yoneda A, Watanabe T. 2004. Effects of oxygen concentration and antioxidants on the in vitro developmental ability, production of reactive oxygen species (ROS), and DNA fragmentation in porcine embryos. Theriogenology, 62:1186-1197.

Li MW, Yudin AI, VandeVoort CA, Sabeur K, Primakoff P, Overstreet JW. 1997. Inhibition of monkey sperm hyaluronidase activity and heterologous cumulus penetration by flavonoids. Biol Reprod, 56:1383-1389.

Li Y, Zhang ZZ, He CJ, Zhu KF, Xu ZY, Ma T, Tao JL, Liu GS. 2015. Melatonin protects porcine oocyte in vitro maturation from heat stress. J Pineal Res, 59:365375.

Park HJ, Park YJ, Kim JW, Yang SG, Jung JM, Kim MJ, Kang MJ, Cho YH, Wee G, Yang HY, Song BS, Kim SU, Koo DB. 2017. Melatonin improves the meiotic maturation of porcine oocytes by reducing endoplasmic reticulum stress during in vitro maturation. $J$ Pineal Res, 64(2). doi: 10.1111/jpl.12458.

Petters RM, Wells CD. 1993. Culture of pig embryos. J Reprod Fertil Suppl, 48:61-73.

Pohanka M. 2011. Alzheimer's disease and related neurodegenerative disorders: implication and counteracting of melatonin. J Appl Biomed, 9:185-196.

Reiter RJ, Manchester LC, Tan DX. 2010. Neurotoxins: free radical mechanisms and melatonin protection. Curr Neuropharmacol, 8:194-210.

Salehi M, Kato Y, Tsunoda Y. 2014. Effect of melatonin treatment on developmental potential of somatic cell nuclear-transferred mouse oocytes in vitro. Zygote, 22:213-217.

Shi JM, Tian XZ, Zhou GB, Wang L, Gao C, Zhu SW, Zeng SM, Tian JH, Liu GS. 2009. Melatonin exists in porcine follicular fluid and improves in vitro maturation and parthenogenetic development of porcine oocytes. J Pineal Res, 47:318-323.

Tatemoto H, Tokeshi I, Nakamura S, Muto N, Nakada T. 2006. Inhibition of boar sperm hyaluronidase activity by tannic acid reduces polyspermy during in vitro fertilization of porcine oocytes. Zygote, 14:275-285.

Tokeshi I, Yoshimoto T, Muto N, Nakamura S, Ashizawa K, Nakada T, Tatemoto H. 2007. Antihyaluronidase action of ellagic acid effectively prevents polyspermy as a result of suppression of the acrosome reaction induced by sperm-zona interaction during in vitro fertilization of porcine oocytes. $J$ Reprod Dev, 53:755-764.

Wang WH, Day BN, Wu GM. 2003. How does polyspermy happen in mammalian oocytes? Microsc Res Tech, 61:335-341.

Wei D, Zhang C, Xie J, Song X, Yin B, Liu Q, Hu L, Hao H, Geng J, Wang P. 2013. Supplementation with low concentrations of melatonin improves nuclear maturation of human oocytes in vitro. J Assist Reprod Genet, 30:933-938.

Whitaker BD, Knight JW. 2004. Exogenous $\gamma$ glutamyl cycle compounds supplemented to in vitro maturation medium influence in vitro fertilization, culture, and viability of porcine oocytes and embryos. Theriogenology, 62:311-322. 